\title{
Correction of Homogenized Lamination Stacks via a Subproblem Finite Element Method
}

\author{
*Patrick Dular, ${ }^{\dagger}$ Mauricio V. Ferreira da Luz, ${ }^{\dagger}$ Patrick Kuo-Peng and ${ }^{\circ}$ Laurent Krähenbühl \\ *University of Liege, ACE, Belgium "Universidade Federal de Santa Catarina, GRUCAD, Brazil \\ Univeristé de Lyon, Ampère, France \\ E-mail: Patrick.Dular@ulg.ac.be
}

\begin{abstract}
A subproblem finite element method is developed for efficient modeling of lamination stacks in magnetic cores. Their homogenization is first performed followed by local corrections in certain thin regions separately, surrounded by their insulating layers and the remaining thin regions kept homogenized. Accurate determinations of Joule losses are obtained.
\end{abstract}

Index Terms — Finite element method, homogenization, lamination stack, subproblems.

\section{INTRODUCTION}

The consideration of lamination stacks in finite element (FE) analyses is a source of difficulty regarding the mesh as well as the numerical solving. For numerous laminations of a magnetic core, separated with insulating layers, their homogenization is usually the only feasible solution for a 3-D FE analysis [1].

Homogenization models are nevertheless tainted with errors, in particular on the border of the homogenized domains. It is here proposed to perform local corrections of homogenized solutions via a subproblem (SP) FE method [2]. The correction SPs use sources calculated from the homogenized solution and allows local corrections of the fields and current densities in regions of interests, allowing to improve global quantities as well such as Joule losses, resistances and inductances.

\section{Progressive Models - Methodology}

Homogenization model- To avoid the explicit definition of all the laminations of a magnetic core, the laminated region is first converted into a continuum $\Omega_{l s, 1}$ in which the eddy currents produced by both parallel and perpendicular fluxes are taken into account with adequate terms in the weak FE formulation [1]. The homogenization method considers the analytical solution of the equations governing the eddy currents in each lamination and averages the actual magnetic flux and current densities along the thickness (Fig. 1a).

Correction of homogenization model- A set of corrections are locally done for one or several laminations (Figs. 1b-d), at some particular positions, e.g., where significant edge effects occur. The chosen thin regions for each correction are now defined with their actual volumes $\Omega_{c, 2}$ surrounded by their insulating layers $\Omega_{n c, 2}$, defining $\Omega_{v o l, 2}=\Omega_{c, 2} \cup \Omega_{n c, 2}$, that can be finely meshed, and the other thin regions kept homogenized, coarsely meshed. The correction solution is calculated via an SP [2] with adequate sources. The key is to suppress the homogenized solution in $\Omega_{v o l, 2}$ while keeping it unchanged outside, simultaneously with the consideration of the actual permeabilities and conductivities in $\Omega_{v o l, 2}$. This is done by defining both normal and tangential trace discontinuities of the correction field through the boundary of $\Omega_{v o l, 2}$ as SSs equal to the opposite of the corresponding traces of the homogenized field in $\Omega_{l s, 1}$ along the fictitious boundary of $\Omega_{v o l, 2}$ there. This requires a mesh projection in a layer of FEs along the boundary of $\Omega_{v o l, 2}$. Each volume correction thus gives the total field in $\Omega_{v o l, 2}$ (Figs. 1b-d), is discontinuous through its boundary and quickly decreases outside, which justifies the use of a coarse mesh in the outer region.

After corrections, significant accuracy improvements (tens of \%) are obtained in a large frequency range (Fig. 2). It has been checked that the corrected solutions are similar to the heavy full solutions with an error easily lower than $1 \%$. A very good accuracy is obtained even when correcting each lamination separately (Fig. 1b) or in small groups (Fig. 1c). Details will be given on the SP formulation and the advantages of the method will be discussed.

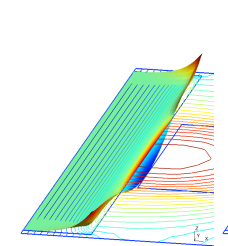

(a)

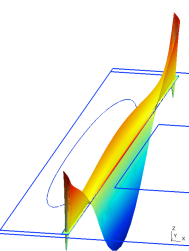

(b)

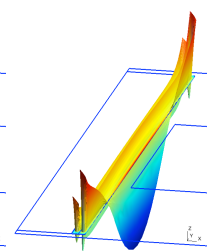

(c)

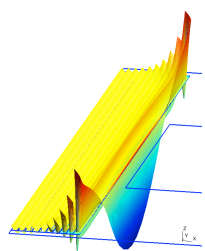

(d)
Fig. 1. Lamination stack (half core, number of lam. $=10$, lam. thickness $0.5 \mathrm{~mm}$, insulation $20 \mu \mathrm{m}, \mu_{\mathrm{r}, \mathrm{lam}}=2500, \sigma_{\mathrm{lam}}=510^{6} \mathrm{~S} / \mathrm{m}$; with air gaps, for longitudinal and transverse fluxes): homogenized solution (a) and corrected solutions in 1 (b), 2 (c) and all laminations (d) (frequency $1 \mathrm{kHz}$ ) (field lines; eddy current density in elevation).
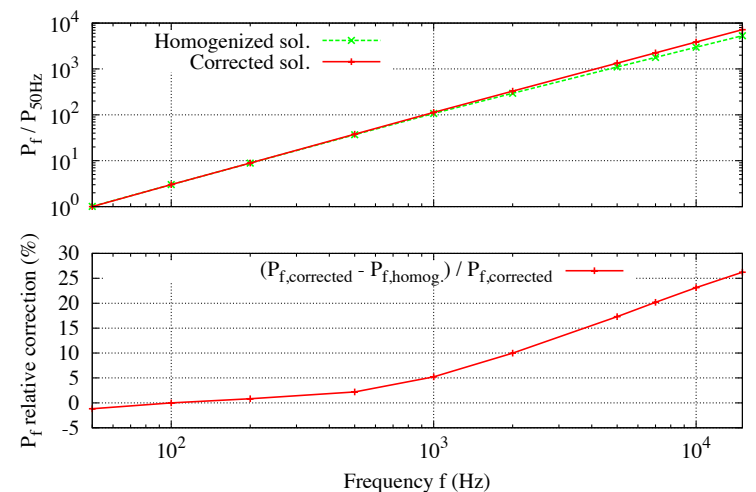

Fig. 2. Joule losses in lamination stack versus frequency $f$ for homogenized and corrected solutions: ratio (in log scale) with losses at $50 \mathrm{~Hz}$ (top), relative correction strongly increasing with $f$ (bottom).

\section{REFERENCES}

[1] L. Krähenbühl, P. Dular, T. Zeidan and F. Buret. "Homogenization of lamination stacks in linear magnetodynamics", IEEE Trans. Magn., vol. 40, no. 2, pp. 912-915, 2004

[2] P. Dular, L. Krähenbühl, R.V. Sabariego, M. V. Ferreira da Luz, P. Kuo-Peng and C. Geuzaine. "A finite element subproblem method for position change conductor systems", IEEE Trans. Magn., vol. 48, no. 2, pp. 403-406, 2012. 